\title{
Nonelectrostatic Ionic Forces between Dissimilar Surfaces: A Mechanism for Colloid Separation
}

\author{
Drew F. Parsons* and Barry W. Ninham \\ Research School of Physical Sciences and Engineering, Australian National University, Canberra, ACT 0200, Australia
}

Supporting Information

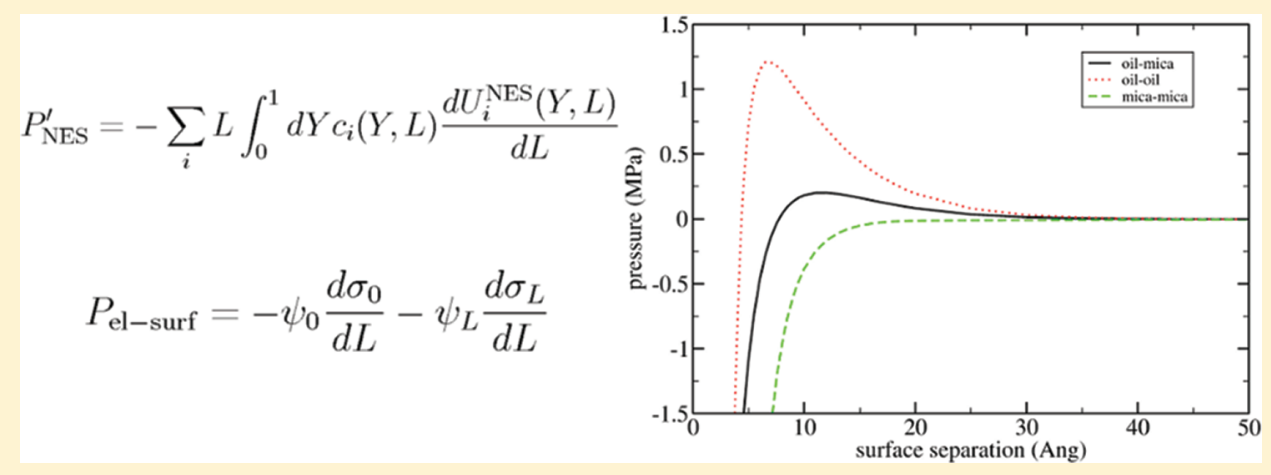

ABSTRACT: The interaction between two dissimilar surfaces across an electrolyte is re-examined. The focus is on effects of ionspecific dispersion forces missing from classical electrostatic double-layer theory. The pressure between two flat surfaces is derived by two alternate methods (midpoint and whole domain approaches). Significant differences emerge from expectations of classical theory. These are illustrated by model interactions across electrolytes of mica and oil-like surfaces. A novel consequence that emerges from inclusion of ionic dispersion forces is the possible separation of mixed colloidal suspensions at moderate $(0.1 \mathrm{M})$ concentrations of divalent salt. Repulsion between the model oil and mica surfaces is found to be due to entropic repulsion driven by high adsorption of both counterions and co-ions at the mica surface. Co-ion adsorption is a consequence of electric field reversal ("charge reversal"), caused by attractive ionic dispersion interactions of the counterion to the mica surface. Charge reversal is also found with monovalent electrolyte but only at impracticably high concentrations.

\section{INTRODUCTION}

Heterocoagulation, involving interactions between dissimilar surfaces, is a problem of wide application. ${ }^{1-6}$ With symmetric systems of identical surfaces, a large volume of recent literature ${ }^{7-11}$ has shown that the inclusion of ionic dispersion forces is necessary to deal with Hofmeister effects or ion specificity of interactions. With dissimilar or asymmetrically charged particle interactions, ${ }^{2,12-17}$ no work has been done to address such contributions. We do so here.

The background to the problem can be seen in several recent summaries on the current level of understanding of ion specific effects $^{7,18}$ and the matter has also been addressed by others. ${ }^{19}$

In section 2 we derive the pressure between two dissimilar flat surfaces, expressed in the same form used previously in the literature (midpoint formula). In particular we derive the component due to ionic dispersion in an asymmetric system and identify explicitly an additional electrostatic surface contribution. We discuss the independence of the total pressure from the position of the midpoint using a nonelectrolytic glucose solution as an example.

In section 2.3 we present an alternative derivation using the whole domain between the two surfaces which yields the same total pressure. This whole domain formula is required for more sophisticated models of ionic interactions where the nonelectrostatic ionic interactions are not separable into distinct interactions with each surface.

An important possible consequence of the new results emerges in illustrative calculations of model oil-like and mica surfaces in section 3 . It seems that ionic dispersion forces in divalent salt at moderate $0.1 \mathrm{M}$ concentrations may assist in the separation of mixed colloidal suspensions. In this section we also discuss the relative contributions of each component to the total pressure. Repulsion between the model oil and mica surfaces is found to be due to entropic repulsion driven by high surface adsorption of both counterions and co-ions. Co-ion adsorption is a consequence of electric field reversal ("charge reversal"), caused by ionic dispersion interactions of the counterion.

Finally in section 3.3 we show that field reversal can even occur in monovalent salt, but only at impracticably high concentrations above $1 \mathrm{M}$.

Received: December 16, 2011

Revised: March 6, 2012

Published: March 8, 2012 


\section{FREE ENERGY AND DERIVATION OF THE DISJOINING PRESSURE}

2.1. Total Free Energy. The total free energy of the system (omitting the direct surface-surface van der Waals dispersion interaction, or Hamaker energy) is ${ }^{20,21}$

$$
F=F_{\text {en }}+F_{\text {el }}+F_{\mathrm{NES}}+F_{\text {chem }}
$$

We do not consider here ion-ion interactions (as addressed, e.g., by modified Poisson-Boltzmann methods ${ }^{22}$ or by integral equation methods ${ }^{23,24}$ ). Nor do we consider electrostatic image forces. ${ }^{25-27}$ Both these effects are neglected in, and are a limitation of, the mean field Poisson-Boltzmann model developed below. Image forces usually-depending on dielectric constantprovide a repulsive contribution to surface forces ${ }^{26}$ and play a role in the surface tension of electrolyte solutions. ${ }^{27} \mathrm{~A}$ further subtlety arises due to screening of the image forces. ${ }^{28}$ We omit these subtleties in order to allow us to focus on the derivation of the contribution of nonelectrostatic (ionic dispersion) forces to the total disjoining pressure and to give an idea of the size of such contributions.

The entropic contribution is ${ }^{21}$

$$
F_{\mathrm{en}}=k T \sum_{i} \int_{0}^{L} d z\left\{c_{i}(z) \ln \frac{c_{i}(z)}{c_{i 0}}-c_{i}(z)+c_{i 0}\right\}
$$

$c_{i 0}$ is the bulk activity of ion $i$. Note that to be strictly rigorous, throughout this paper the variable $c$ refers to ion activity. However for simplicity we take activity coefficients equal to unity, identifying ion activity with ion concentration. So our results, although consistent with the usual electrostatic theory do not deal with ion correlations. Our illustrative calculations should therefore be taken as indicative rather than quantitative.

The electrostatic energy is derived from the electrostatic potential $\psi$ due to the electrolytic and surface charges

$$
F_{\mathrm{el}}=\frac{1}{2} \int D E=\frac{\varepsilon_{0} \varepsilon}{2} \int_{0}^{L}\left(\frac{d \psi}{d z}\right)^{2} d z
$$

$L$ is the separation between the two surfaces and marks the positions of the actual surfaces where the intrinsic surface charges lie. This is distinct from the position of any Stern layer, which is a layer of charge due to adsorbed ions that may be displaced from the surface due to distances of closest approach of ions with finite size. This electrostatic energy is calculated from the electrostatic potential $\psi$, which in turn is calculated from ionic charge distributions $q_{i} c_{i}(z)$ via the Poisson equation (for a planar system)

$$
\varepsilon_{0} \varepsilon \frac{d^{2} \psi(z)}{d z^{2}}=-\sum_{i} q_{i} c_{i}(z)
$$

The ion concentration profiles $c_{i}(z)$ are Boltzmann distributions which could be inferred experimentally, ${ }^{29-32}$ or calculated by computer simulation, ${ }^{32-36}$ by integral equation methods ${ }^{23,25,37}$ or the Poisson-Boltzmann method ${ }^{38,39}$ (including nonelectrostatic ion interactions) or calculated by hybrid methods. ${ }^{40-42}$ In this paper we employ the Poisson-Boltzmann method which solves for $c_{i}(z)$ self-consistently and nonlinearly with the Poisson equation using the Boltzmann distribution

$$
c_{i}(z)=c_{i 0} \exp \left[-\left(q_{i} \psi(z)+U_{i}^{\mathrm{NES}}(z, L)\right) / k T\right]
$$

$c_{i 0}$ is the bulk concentration of ion $i . U_{i}^{\mathrm{NES}}(z, L)$ describes the nonelectrostatic contributions to the chemical potential (interaction potential) of the ion. These nonelectrostatic interactions perturb the Boltzmann distribution away from that which would occur purely through electrostatic interactions. ${ }^{28}$

The nonelectrostatic component to the free energy, $F_{\mathrm{NES}}$, is given by the interaction $U_{i}^{\mathrm{NES}}(z, L)$ of ion $i$ at perpendicular position $z$ between the two surfaces

$$
F_{\mathrm{NES}}=\sum_{i} \int_{0}^{L} d z c_{i}(z) U_{i}^{\mathrm{NES}}(z, L)
$$

$c_{i}(z)$ is the concentration (activity) of ion $i$ at $z$. The main focus of this paper is to derive the component to the disjoining pressure corresponding to this term, but for the case of dissimilar surfaces. We take the nonelectrostatic interaction to be an ionic dispersion interaction (see eq 17 below), but the formalism applies to any nonelectrostatic interaction conforming to eq 6. For instance the distance of closest approach of an ion to a surface can be handled via additional nonelectrostatic hard sphere interactions (which may include hydration of the surfaces as well as the ions ${ }^{8}$ ) using the same formalism. Likewise image interaction energies (including screened image interactions), although electrostatic in origin, may be added using the same formal set of equations represented by eq 6 or the corresponding pressure terms derived below, by setting $U_{i}^{\mathrm{NES}}$ to the relevant image energy. A form of ion-ion repulsive core interaction can be modeled as a nonelectrostatic steric energy that restrains attractive ion interactions, with the effect of capping concentrations within physical limits. ${ }^{9}$

The final term $F_{\text {chem }}$ describes the change in free energy due to an equilibrium between ions in solution and on the surface (chemisorption or dissociation), with an exchange of ions taking place to balance the chemical potentials of surface and solution. This kind of equilibrium is relevant to constant surface potential (Nernstian) conditions or to charge-regulated systems. ${ }^{17,43-46}$ In the examples presented in this paper, however, for the sake of simplicity we adopt constant surface charge conditions so that $F_{\text {chem }}$ is constant. We include it here for completeness and to address a term arising from the electrostatic component in the course of the derivation of the disjoining pressure (eq 10).

Ionic nonelectrostatic interactions influence the total free energy in two ways. Their direct contribution is given by $F_{\mathrm{NES}}$ via the ionic nonelectrostatic interaction $U_{i}^{\mathrm{NES}}(z, L)$ (e.g., ionic dispersion). They also have an indirect influence by altering the ion concentration profiles $c_{i}(z)$ from those determined by electrostatics alone and therefore the electric field. In this sense the electrostatic and nonelectrostatic components are not wholly separable from one another, but rather have an (indirect) influence on each other.

The disjoining pressure is derived from the free energy of the system, $P=-d F / d L$. In some studies the derivative has been taken numerically. ${ }^{39,41}$ We follow the approach ${ }^{20,22}$ of deriving a formula to calculate the disjoining pressure analytically.

2.2. Disjoining Pressure: Midplane Approach. We calculate the pressure between the two surfaces by applying the variational approach of Burak and Andelman ${ }^{22}$ and of Edwards and Williams. ${ }^{20}$ The variation $\delta F$ of the free energy is determined through insertion of a midplane at point $z_{m}$ between the two surfaces, which need not necessarily be the strict equidistant midpoint at $L / 2$. Technical details are given in the Supporting Information. 
The electrolytic disjoining pressure $P=-d F / d L$ (not including the surface-surface Hamaker contribution) is

$$
P\left(L \mid z_{m}\right)=P_{\mathrm{en}}+P_{\mathrm{el}-\mathrm{mid}}+P_{\mathrm{el}-\text { surf }}+P_{\mathrm{NES}}+P_{\text {chem }}
$$

where the entropic contribution $P_{\text {en }}$ includes the subtraction of the bulk external pressure $P_{0}$

$$
P_{\mathrm{en}}=k T \sum_{i}\left[c_{i}\left(z_{m}\right)-c_{i 0}\right]
$$

The electrostatic contribution is split into a midplane term

$$
P_{\mathrm{el}-\text { mid }}=-\frac{\varepsilon_{0} \varepsilon}{2}\left(\frac{d \psi}{d z}\right)_{z_{m}}^{2}
$$

and a surface term describing the rate of change of surface charge as separation varies

$$
P_{\text {el-surf }}=-\psi(0) \frac{d \sigma_{0}}{d L}-\psi(L) \frac{d \sigma_{L}}{d L}
$$

The general relationship between $P_{\text {chem }}$ and $P_{\text {el-surf }}$ is addressed below. Here $P_{\text {chem }}=0$ (no ion exchange between surface and solution occurs).

To derive the nonelectrostatic component $P_{\mathrm{NES}}$, the midplane variational approach requires the nonelectrostatic energy of an individual ion to be decomposed into its interactions with each surface

$$
U_{i}^{\mathrm{NES}}(z, L)=U_{1 i}(z)+U_{2 i}(L-z)
$$

$U_{1 i}$ is the nonelectrostatic interaction with the left surface located at $z=0$ and $U_{2 i}$ is the nonelectrostatic interaction with the right surface at $z=L$ (we dropped the NES label for convenience). The nonelectrostatic (ion dispersion) contribution, then, is

$$
\begin{aligned}
P_{\mathrm{NES}}= & -\sum_{i}\left[\int_{0}^{z_{m}} d z c_{i}(z) U^{\prime}{ }_{2 i}(L-z)\right. \\
& \left.+\int_{z_{m}}^{L} d z c_{i}(z) U^{\prime}{ }_{1 i}(z)\right]
\end{aligned}
$$

where $U_{j i}^{\prime}(x)$ refers to the derivative (with respect to $x$ ) of the nonelectrostatic interaction $U_{j i}(x)$ of ion $i$ at a distance $x$ from surface $j$.

In eq 12 three points need to be emphasized. First, the formula generalizes the result of Edwards and Williams derived for two similar surfaces. ${ }^{20}$ This point is the chief focus of the current paper.

Second, the electrostatic contribution $P_{\text {el-mid }}+P_{\text {el-surf }}$ is always negative; hence this contribution is always attractive. Under conditions of positive adsorption the entropic contribution $P_{\mathrm{en}}$ is positive, i.e., repulsive. The nonelectrostatic contribution $P_{\mathrm{NES}}$ may be attractive or repulsive depending on the specific ions and concentration and less important at low concentrations. It follows that repulsive interactions are largely entropic, not electrostatic, in origin. (A semantic point: the literature often refers to "electrostatic repulsion". ${ }^{47-52}$ Taken literally, this phrase is wrong. The misconception occurs since the ion concentration profiles responsible for the entropic term are driven partly by ionic electrostatic interactions. This point is certainly known, ${ }^{53}$ but we fear that physical intuition into the behavior of the system is obfuscated by use of the phrase "electrostatic repulsion". It tends to draw a casual observer's gaze toward the charge of the electrolyte, when the charge profile is perhaps more important. A counter example can be made by considering a nonelectrolyte, such as a sugar solution. In this case electrostatic interactions are zero but the entropic contribution still appears (and is still repulsive) because of molecular concentration profiles driven by nonelectrostatic forces (molecular dispersion forces). The term "entropic repulsion" is more appropriate.)

Third, and importantly, we make the following observations. We have included the electrostatic surface term $P_{\text {el-surf }}$ eq 10 , which describes the contribution of a surface charge that varies as the separation between walls varies. The term is zero under constant surface charge conditions, but under other conditions, namely, constant potential (Nernstian) or charge regulated conditions ${ }^{17,54,55}$ where the surface charge varies with separation, it generally provides a strongly attractive force. Under these varying charge conditions it is counterbalanced by the chemical term $P_{\text {chem }}$ which is generally repulsive. $P_{\text {el-surf }}$ and $P_{\text {chem }}$ cancel exactly under Nernstian constant potential conditions. In charge-regulated systems $P_{\text {chem }}$ typically contains additional repulsion attributable to the varying surface potential, although there are further subtleties depending on whether the charge regulated system is "sub-Nernstian" or "superNernstian", 46,56 and whether the nonelectrostatic component of the chemical potential of the charged surface site depends on the number of particles transferred. ${ }^{44,45}$ We leave further discussion of the role of the chemical term for elsewhere. For simplicity we apply conditions of constant surface charge in the numerical illustrations in this paper.

2.2.1. Midplane Dependence of the Pressure. At this point, prior to considering dispersion contributions, we rehearse the nature of the pressure components in the classical electrostatic case. The original derivations of the disjoining pressure were obtained by balancing hydrostatic pressure against the stress of the electrostatic field. ${ }^{57}$ Under hydrostatic conditions the pressure was independent of the position $z_{m}$ taken between the two surfaces. The midpoint $z_{m}=L / 2$ was therefore taken for convenience, where the electrostatic stress was zero in a symmetric system. The independence of the disjoining pressure $P\left(L \mid z_{m}\right)$ from the position of $z_{m}$ for a purely electrostatic system without ionic dispersion is illustrated in Figure 1 for fixed

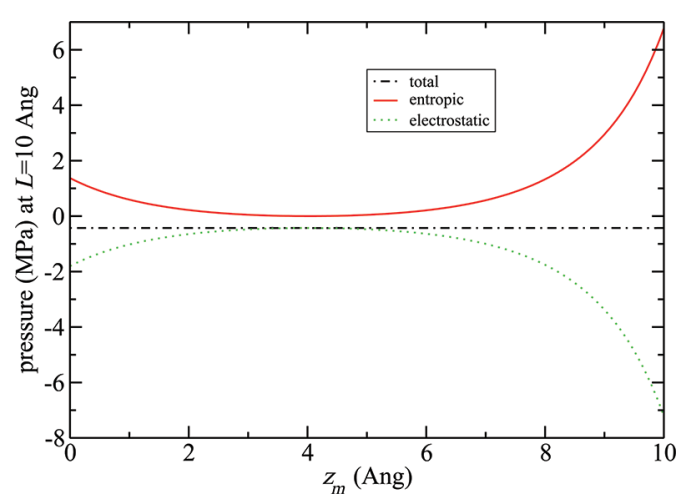

Figure 1. Disjoining pressure $P\left(L=10 \mid z_{m}\right.$ ), eq 7 (excluding the Hamaker pressure) of $0.1 \mathrm{M} \mathrm{MgCl}_{2}$ between two surfaces at fixed separation $10 \AA$ as a function of the position of the midpoint $z_{m}$. Ionic dispersion is switched off $\left(P_{\mathrm{NES}}=0\right)$. Asymmetric constant charge is used, $\sigma_{0}=+0.05$ and $\sigma_{0}=-0.1 \mathrm{Cm}^{-2}$ on left and right-hand surfaces, respectively. Entropic and electrostatic components are shown to illustrate dependence on $z_{m}$. The total disjoining pressure is -1.10 $\mathrm{MPa}$, independent of $z_{m}$. 
separation $L=10 \AA$. As $z_{m}$ is varied away from $L / 2$, the relative strengths of the entropic $\left(P_{\text {en }}\right)$ and electrostatic $\left(P_{\text {el-mid }}\right)$ components varies. But the two components are coupled such that their $z_{m}$ dependence cancels. The total pressure is independent of $z_{m}$.

2.2.2. Nonelectrolytic Solution and $z_{m}$. By contrast, we now consider the example of a purely nonelectrostatic system constructed by taking a $1 \mathrm{M}$ glucose solution between surfaces of tetradecane and polystyrene. We apply a molecular dispersion interaction described in more detail below, ${ }^{17}$ with dispersion coefficients $B_{C_{14}}=1.19 \times 10^{-50} \mathrm{~J} \mathrm{~m}^{3}$, repulsive against tetradecane, and $B_{\mathrm{PS}}=-2.57 \times 10^{-50} \mathrm{~J} \mathrm{~m}^{3}$, attractive against polystyrene. These dispersion coefficients were calculated using procedures described previously ${ }^{58}$ (taking the nonretarded interaction for simplicity), with the dynamic polarizability $\alpha(i \omega)$ of $\mathrm{D}$ - $\beta$-glucose calculated by DFT methods with a PBE0 functional $^{59}$ and an aug-cc-pVDZ basis set. ${ }^{60}$ The radius of the glucose molecule (hard sphere radius, determined from ab initio volume calculations ${ }^{61}$ ) was $3.22 \AA$. The dielectric functions of Parsegian and Weiss ${ }^{62}$ were used for the two surfaces.

When taking a nonelectrostatic potential of the form $1 / x^{n}$ ( $n=3$ in the case of molecular dispersion interactions, see eq 17), an analytic expression for the disjoining pressure is not available if a Boltzmann distribution is maintained. (If a non-Boltzmann distribution is applied, for instance, imposing $c(z) \equiv c_{0}$ at all $z$, thereby making $P_{\mathrm{en}}=0$, then a $z_{m}$-dependence will appear.) Instead, we plot in Figure 2 the disjoining pressure (for a

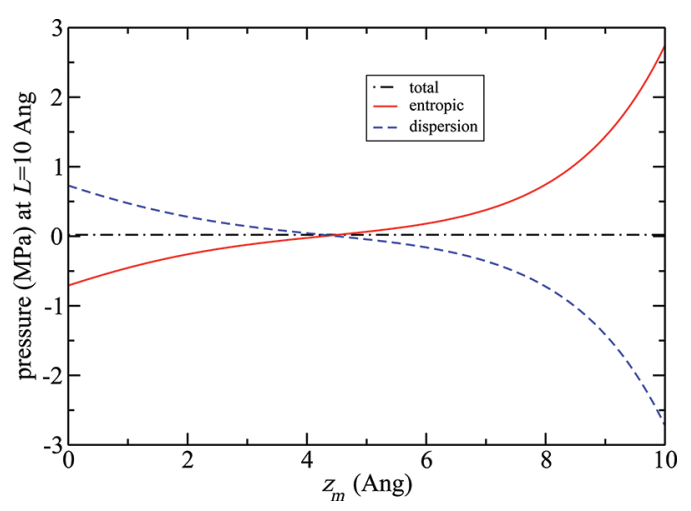

Figure 2. Disjoining pressure $P\left(L \mid z_{m}\right)$ (excluding the Hamaker pressure) of $1 \mathrm{M}$ glucose solution between tetradecane and polystyrene surfaces at fixed separation $L=10 \AA$, as a function of the position of the midpoint $z_{m}$. No electrostatic interactions are present, glucose molecules have dispersion interactions with each wall. Entropic and nonelectrostatic components are shown to illustrate dependence on $z_{m}$. The total disjoining pressure is $0.0207 \mathrm{MPa}$, independent of $z_{m}$.

Boltzmann system) as a function of $z_{m}$ between two surfaces at a fixed separations $L=10 \AA$. This calculation should be understood as illustrative; in a real nonelectrolytic solution at high concentrations the molecule is likely to also be subject to short-range oscillatory forces (solute-solute and solutesolvent correlations) similar to those found in electrolytes. ${ }^{40,42}$

We see that the total pressure $P\left(L \mid z_{m}\right)$ calculated via middle point $z_{m}$ is constant, independent of the position of $z_{m}$. We conclude that the midpoint formulation of the disjoining pressure including nonelectrostatic interactions, $P\left(L \mid z_{m}\right)$ from eq 7 , is reliable. Moreover we next confirm the correctness of the formula by presenting an alternate formulation for the same total disjoining pressure.
2.3. Disjoining Pressure: Whole-Domain Approach. The traditional formulation of the disjoining pressure (eqs 7 and 8) involves terms defined at midpoint $z_{m}$. This arose through the variational technique used, in which a slab of thickness $\delta L$ was inserted at $z_{m}$. An alternate formulation of the pressure may be made by spreading the variation $\delta L$ of separation $L$ across the entire region $z \in[0, L]$. This is particularly relevant for more sophisticated models of ionic interactions where the nonelectrostatic interaction $U_{i}^{\mathrm{NES}}$ is not separable into distinct interactions with each surface, as assumed in eq 11. For instance in one nonseparable case ${ }^{63,64}$ we have a kind of three-body effect where the dispersion interaction of an ion at point $z$ with the left wall is perturbed nonlinearly due to the presence of the second wall at point $z$. Another example is steric repulsion, which is a repulsive interaction that would prevent ion concentrations from exceeding a certain cap. Such a steric repulsion would depend nonlinearly on the local ion concentration. In these scenarios we cannot separate the variation of the ionic dispersion energy into two separate derivatives against opposing walls, as done in eq 12, and the midpoint formula therefore cannot be applied. Instead the derivative $d U_{i}^{\mathrm{NES}}(z, L) / d L$ needs to be taken as a whole quantity, over the whole domain of $z$ between 0 and $L$. The alternate whole domain formula is also useful as a numerical check to verify that a midpoint calculation has been implemented correctly.

The $d / d L$ derivative is made well-defined, removing the ambiguity of the $L$-dependence of $z$, by renormalizing the domain of $U_{i}^{\mathrm{NES}}(z, L)$ such that the two variables are independent. That is, we take $U_{i}^{\mathrm{NES}}(Y, L)$, defining $Y=z / L$ with $Y \in[0,1] . d U_{i}^{\mathrm{NES}}(\mathrm{Y}, \mathrm{L}) / d L$ is then unambiguously defined. Integrals $\int_{0}^{L} d z$ are replaced by $L \int_{0}^{1} d Y$. With this rescaling, the pressure is determined by variation over the whole domain $[0, L]$ (equivalent to the standard derivative $P=-d F / d L$ ). The derivation is given in detail in the Supporting Information; the resulting pressure is

$$
P=P_{\text {en }}^{\prime}+P_{\text {el-surf }}+P_{\text {el-ion }}^{\prime}+P_{\text {NES }}^{\prime}+P_{\text {chem }}
$$

This total disjoining pressure $P$ is equal to the total pressure $P\left(L \mid z_{m}\right)$ calculated by the midplane formula, eq 7 , only the details of some of the components have changed. The nature of the chemical term $P_{\text {chem }}$ was discussed above, it is relevant to systems where the surface charge is in equilibrium with ions in solution (constant potential or charge regulated systems). We consider the constant charge case in the illustrative calculations in this paper, for which $P_{\text {chem }}=0$ (and $\left.P_{\text {el-surf }}=0\right)$. The entropic contribution takes the form.

$$
P_{\text {en }}^{\prime}=k T \sum_{i} \int_{0}^{1} d Y\left[c_{i}(Y, L)-c_{i 0}\right]
$$

The electrostatic contribution due to the ions in solution reduces to

$$
P_{\text {el-ion }}^{\prime}=-\frac{F_{\mathrm{el}}}{L}
$$

where $F_{\mathrm{el}}$ is the total electrostatic free energy, eq 3 .

$P_{\text {el-surf }}$ refers to the same variation in surface charge seen above, $P_{\text {el-surf }}=-\psi_{0} d \sigma_{0} / d L-\psi_{L} d \sigma_{L} / d L$, eq 10 . The nonelectrostatic contribution becomes

$$
P^{\prime}{ }_{\mathrm{NES}}=-\sum_{i} L \int_{0}^{1} d Y_{i}(Y, L) \frac{d U_{i}^{\mathrm{NES}}(Y, L)}{d L}
$$


For the case where the nonelectrostatic interaction $U_{i}^{\mathrm{NES}}$ can be separated into the sum of interactions $U_{j i}(x)$ of ion $i$ with surface $j$, eq 11 , the derivative $d U_{i}^{\mathrm{NES}}(Y, L) / d L=Y U_{1 i}^{\prime}(Y L)+$ $(1-Y) U_{2 i}^{\prime}((1-Y) L)$ is given in terms of a linear combination of simple derivatives $U_{j i}^{\prime}(x)$, (see eq B.1 in the Supporting Information).

Parallels with the midplane formulation of eqs 7 and 8 are clear, with evaluations at $z_{m}$ replaced with integrals over the whole domain. The comparison is not exact however: $P_{\text {en }}^{\prime} \neq$ $P_{\mathrm{en}}$, for instance, and there is a different level of mixing between the different terms (i.e., magnitudes of components are different in the whole-domain formulation compared with the midpoint formulation).

The formulation for the disjoining pressure given here is useful in cases where $d U_{i}^{\mathrm{NES}}(Y, L) / d L$ is not separable into a sum of interactions with each surface. The nonseparable analogue of the dispersion interaction of an ion between two surfaces is known $^{63,64}$ and is considerably more complex than the separated interaction with two walls used in eq 11 with eq 17 . The derivative $d U_{i}^{\mathrm{NES}}(Y, L) / d L$ is not available analytically. The deviation between the separated and nonseparable expressions will become increasingly large as the separation between surfaces becomes small. However in practice that deviation is small: testing the full nonseparable expression for the example ions of $\mathrm{Br}^{-}$between two mica surfaces, the deviation between separated and nonseparable expression is less than $1 \%$ at a separation of $5 \AA$. The expressions given here are important chiefly because they remove the ambiguity of the influence of the position of the midpoint coordinate $z_{m}$ discussed in the previous sections.

\section{NUMERICAL ILLUSTRATION: COLLOID SEPARATION BY DIVALENT SALT}

As an example of the application of the new formulas, we present an example for the case of a model $\mathrm{MgCl}_{2}$ electrolyte between an oil-like surface with fixed surface charge +0.05 $\mathrm{Cm}^{-2}$ and a mica surface with fixed surface charge $-0.1 \mathrm{Cm}^{-2}$. The surface charges have been selected artificially to emphasize the asymmetry between the surfaces. Magnitudes are therefore exaggerated but otherwise physically correspond to the surface charges of some crude oils in an acidic electrolyte around $\mathrm{pH} \mathrm{3-4.}{ }^{65}$

The dispersion interactions between surface $j$ and ion $i$ at a distance $x$ from the surface are taken to $\mathrm{be}^{58}$ (only the nonretarded interaction is dealt with here)

$$
U_{d i j}(x)=\frac{B_{i j}}{x^{3}} g(x)
$$

with $\mathrm{g}(x)=1+\left(2 x /(\pi)^{1 / 2} a_{i}\right)\left(2 x^{2} / a_{i}^{2}-1\right) \exp \left[-x^{2} / \mathrm{a}_{i}^{2}\right]-(1+$ $\left.4 x^{4} / a_{i}^{4}\right) \operatorname{erfc}\left[x / a_{i}\right]$. This kind of nonelectrostatic potential has previously been used in symmetric systems (identical surfaces) by Boström and ourselves ${ }^{66-70}$ and others ${ }^{39,71,72}$ for symmetric systems, in some cases with the simplification $g(x) \equiv 1$ together with a cutoff distance. Others have calculated a nonelectrostatic potential of mean force from computer simulation, used then in a Poisson-Boltzmann calculation. ${ }^{40,41}$

The corresponding derivative, used in eq 12 , is

$$
U^{\prime}{ }_{d i j}(x)=-\frac{3 B_{i j}}{x^{4}} h(x)
$$

where

$$
\begin{aligned}
h(x)= & 1-\frac{2 x}{\sqrt{\pi} a_{i}} e^{-x^{2} / a_{i}^{2}}\left(1+\frac{2 x^{2}}{3 a_{i}^{2}}\right)-\left(1-\frac{4 x^{4}}{3 a_{i}^{4}}\right) \\
& \times \operatorname{erfc}\left(\frac{x}{a_{i}}\right)
\end{aligned}
$$

Dielectric functions for surfaces and water were taken from the literature ${ }^{62,73,74}$ using tetradecane as a model for the positively charged oil-like surface. Ion sizes and ionic polarizabilities were derived from ab initio quantum calculations. ${ }^{58,61} \mathrm{The}^{\mathrm{Mg}^{2+}}$ ion is taken to be strongly hydrated with 10 hydration waters. ${ }^{75}$ The polarizability of the hydration waters is added to that of the cation to give a total polarizability for the whole hydrated ion. ${ }^{76}$ The size of the hydrated ion is set by adding the hard sphere radius of a water molecule to that of the bare ion. ${ }^{61,76}$ The resulting dispersion $B$ coefficients and ion sizes are given in Table 1. The electrostatic potential and ion concentration profiles

Table 1. Ionic Radii ${ }^{61} a$ and Dispersion $B$ Coefficients $\left(10^{-50}\right.$ $\mathrm{Jm}^{3}$ ) of $\mathrm{Mg}^{2+}$ (hydrated), $\mathrm{Cl}^{-}, \mathrm{K}^{+}$, and $\mathrm{I}^{-}$at Mica and OilLike Tetradecane Surfaces

\begin{tabular}{lccc}
\multicolumn{1}{c}{ ion } & $a(\AA)$ & $B($ mica $)$ & $B$ (tetradecane) \\
$\mathrm{Mg}^{2+} \cdot 10 \mathrm{H}_{2} \mathrm{O}$ & 1.54 & -3.6924 & 2.9927 \\
$\mathrm{Cl}^{-}$ & 1.69 & -1.2099 & -0.1472 \\
$\mathrm{~K}^{+}$ & 0.96 & -0.3364 & 0.2961 \\
$\mathrm{I}^{-}$ & 2.12 & -1.9154 & -0.5947 \\
\hline
\end{tabular}

were determined by nonlinear Poisson-Boltzmann calculations. The positively charged oil-like was taken on the left at $z=0$ and negatively charged mica on the right at $z=L$.

The total pressure-distance curve for $0.1 \mathrm{M} \mathrm{MgCl}_{2}$ is shown in Figure 3 (solid black curve). Here we do include the

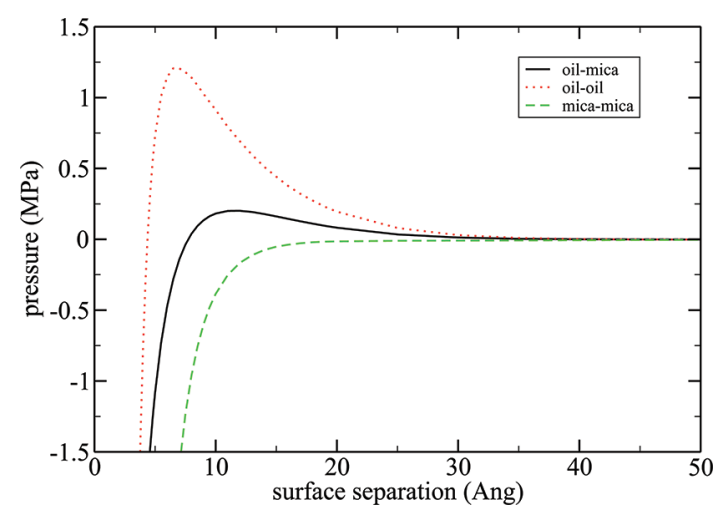

Figure 3. Pressure-distance curves (including the Hamaker pressure) for $0.1 \mathrm{M} \mathrm{MgCl}_{2}$ between an oil-like surface with surface charge $\sigma_{0}=$ $+0.05 \mathrm{Cm}^{-2}$ and mica $\left(\sigma_{0}=-0.1 \mathrm{Cm}^{-2}\right)$. Ion-surface dispersion interactions are included. Mixed phase (oil-mica, solid black) and single phase (oil-oil, dotted red; mica-mica dashed green) interactions are shown.

attractive nonretarded Hamaker (van der Waals) component, $P_{\text {ham }}(L)=-A / 6 \pi L^{3}$, taking an oil-water-mica Hamaker constant $A=1.05 k T$ (calculated using the dielectric functions of the three materials) with $T=25{ }^{\circ} \mathrm{C}$. This figure shows a repulsive oil-mica interaction at a concentration of $0.1 \mathrm{M}$. Interestingly, the interaction is attractive at lower concentrations. The oil-oil and mica-mica interactions in $0.1 \mathrm{M}$ 
$\mathrm{MgCl}_{2}$ are shown in Figure 3 (dotted red and dashed green curves), calculated with Hamaker constants $1.55 k T$ and $2.29 k T$, respectively. The oil colloids are found to be stable (repulsive interactions) in $0.1 \mathrm{M}$ salt. They become attractive at higher concentrations beyond $0.3 \mathrm{M}$, with aggregation of the oil phase setting in. The mica colloids are attractive (will aggregate) at $0.1 \mathrm{M}$ and in fact at all salt concentrations in this model, with the attraction weakest between 0.05 and $1 \mathrm{M}$.

So, in $0.1 \mathrm{M} \mathrm{MgCl}_{2}$ mica-mica interactions are attractive, but oil-oil and oil-mica interactions are repulsive. In this way the calculations suggest that at this optimal concentration the oil-mica mixture may be separated with the mica phase aggregating out of suspension, while the oil-like phase remains in suspension. At lower concentrations oil-mica interactions become attractive, such that the mica phase will aggregate, taking with it a layer of the oil-like phase, producing an unseparated mixed aggregate. At higher concentrations the oiloil interaction becomes attractive, leading again to a mixed aggregate.

This phase separation effect is not predicted in a calculation with electrostatic interactions only, where ionic dispersion interactions are omitted. The oil-mica interaction without ionic dispersion, Figure 4 (solid black curve), is found to be attractive

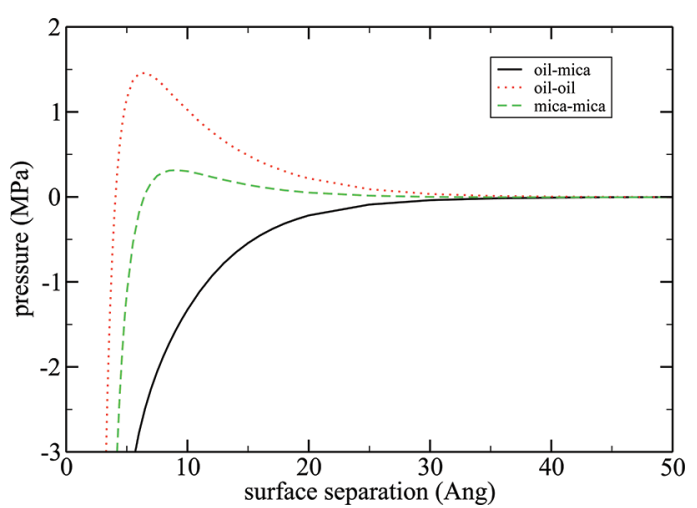

Figure 4. Pressure-distance curves (including the Hamaker pressure) for $0.1 \mathrm{M} \mathrm{MgCl}_{2}$ between an oil-like surface with surface charge $\sigma_{0}=$ +0.05 and mica $\left(\sigma_{0}=-0.1 \mathrm{Cm}^{-2}\right)$. Ion-surface dispersion interactions are not included. Mixed phase (oil-mica, solid black) and single phase (oil-oil, dotted red; mica-mica dashed green) interactions are shown.

(true at all concentrations), inhibiting separation of the two phases. The oil-oil interaction, Figure 4 (dotted red curve), remains qualitatively the same with or without ionic dispersion, repulsive in $0.1 \mathrm{M} \mathrm{MgCl}_{2}$, reflecting the relatively weak dispersion interaction of the $\mathrm{Cl}^{-}$counterion with the oil surface. The mica-mica interaction on the other hand (dashed green curve) is found to be repulsive in the absence of ionic dispersion (similar to oil-oil), in contrast to the attractive interaction found in the presence of ionic dispersion. At higher concentrations both oil-oil and mica-mica interactions become attractive, leading to aggregation, but with no colloid separation. This suggests no separation of the mixed suspension is predicted to take place by a purely electrostatic theory. If aggregation occurs at all, it occurs only as a mixed aggregate.

The separation effect suggested here is ion specific. If the same calculation is performed for $0.1 \mathrm{M} \mathrm{CaCl}_{2}$ (with 7 hydration waters per $\mathrm{Ca}^{2+}$ ion $^{75}$ ), the oil-mica interaction is completely attractive, so separation will not take place. The behavior is probably concentration dependent; that is, $\mathrm{CaCl}_{2}$ will likely exhibit the separation behavior at a higher concentration.

This oil-mica repulsion might help to explain the origin of anomalies (nonadhesion) observed in oil-rock interactions at high concentrations, ${ }^{77,78}$ which cannot be explained by simple Poisson-Boltzmann theory with only electrostatic interactions. Liu and Buckley reported experimental adhesion maps for crude oil on mica. ${ }^{77}$ They found the oil-mica interaction to be nonadhesive at high salt concentrations, consistent with the repulsive peaks that arose due to ionic dispersion forces in Figure 3. Shedid and Ghannam measured a nonmonotonic relationship between salt concentration and the contact angle of a droplet of crude oil on samples of oil-bearing carbonate rock. $^{78}$ The contact angle fell as concentration increased to a critical value around $1 \mathrm{M}$ (50000 ppm salinity) and rose again at higher concentrations. These observations may be consistent with our attractive mica-mica interaction and repulsive oil-oil interaction in the presence of ionic dispersion (Figure 3), if one infers that oil-water and rock-water surface tensions rise or fall with concentration in line with the repulsive or attractive character of the interactions. Shedid and Ghannam's contact angles also showed a second turning point at extreme salinity which likely arises due to higher-order effects not included in a Poisson-Boltzmann description, namely, ion-ion and ionwater correlations. We will address these experimental studies more closely in subsequent work employing a more accurate model of the system (including a regulated surface charge).

3.1. Analysis of Components to Total Pressure. A question to ask in order to understand the separation effect of $0.1 \mathrm{M} \mathrm{MgCl}_{2}$ is why the repulsive peak occurs in the oil-mica interaction. We dissect the various components to the pressure-distance curve in Figure 5, comparing the calculation using the midpoint formula eq 7 (with $z_{m}=L / 2$ ) against the whole domain formula, eq 13 . The total disjoining pressure agrees at all separations with that calculated through the whole domain formula. The total disjoining pressure is the same at all separations in both formulas. The direct dispersion component, eqs 12 and 16, respectively, is found to be repulsive at very small separations (below $5 \AA$ ), but is negligable (midpoint formula) or slightly attractive (whole domain formula) at higher separations where the repulsive peak in the total pressure occurs. Oil-mica repulsion therefore does not take place due to the direct contribution of ionic dispersion interaction. Nor is the repulsion driven by the electrostatic contribution, which is attractive. Rather, both formulas clearly show that repulsion takes place due to the entropic contribution. The two formulas generally differ in the magnitudes of their components: the whole-domain components are each larger in magnitude than their midplane counterparts, indicating a greater degree of cancellation between them. Note however that due to a $z_{m}$ dependence of the pressure components in the midpoint formula (distinct from the total pressure which is independent of $z_{m}$ ), the relationship between the components of the midpoint formula is only relative.

But why is the entropic contribution so strong in the presence of ionic dispersion? In the absence of ionic dispersion the entropic component is still repulsive, but perhaps four times smaller. The reason for the stronger entropic force can be understood from the behavior of the electrostatic potential at the surfaces. The surface potential of each surface as a function of concentration is shown in Figure 6. Electric field reversal (potential reversal) takes place at the mica surface around $0.02 \mathrm{M}$ and is also found at the oil-like surface at high concentrations above $2 \mathrm{M}$. A negative potential is found at the positively 
(a)

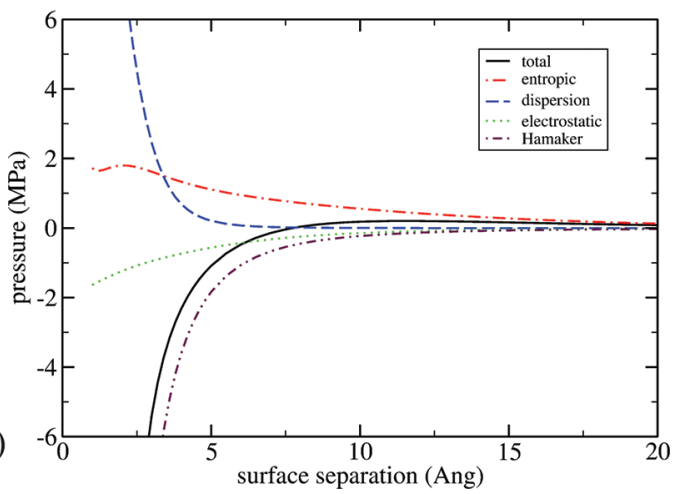

(b)

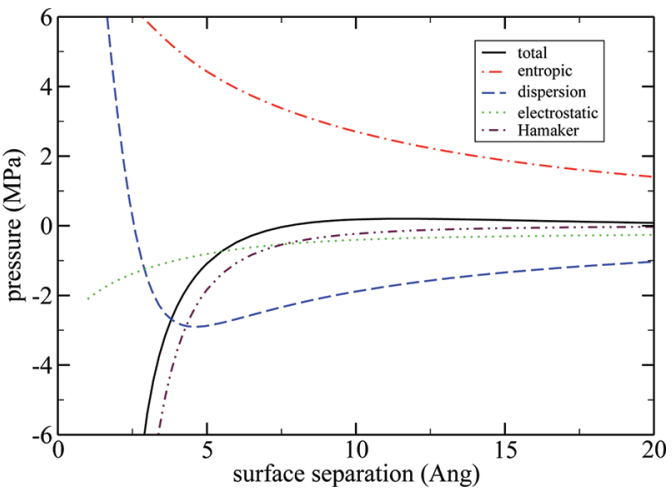

Figure 5. Pressure-distance curves showing components for $0.1 \mathrm{M} \mathrm{MgCl}_{2}$ electrolyte between oil-like surface with surface charge $\sigma_{0}=+0.05$ and mica $\left(\sigma_{0}=-0.1 \mathrm{Cm}^{-2}\right)$. Ion-surface dispersion interactions are included. Alternative formulas are shown: (a) midpoint eq 7, (b) whole-domain eq 13 .

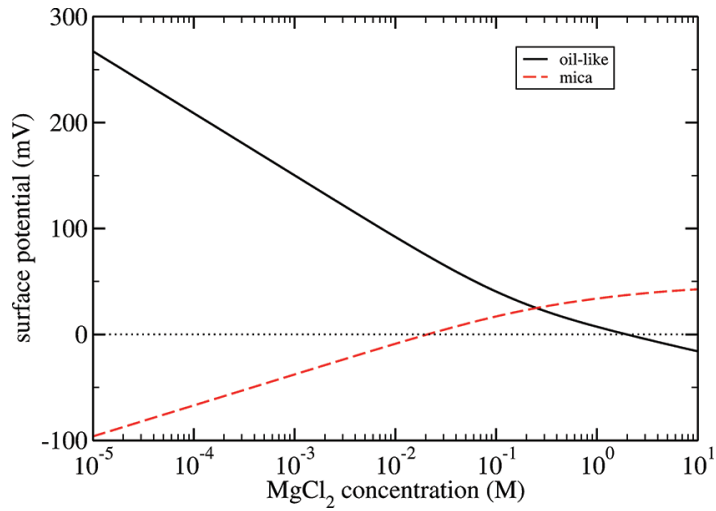

Figure 6. Electrostatic surface potentials of an oil-like surface (surface charge $\left.\sigma_{0}=+0.05\right)$ and mica surface $\left(\sigma_{0}=-0.1 \mathrm{Cm}^{-2}\right)$ in $\mathrm{MgCl}_{2}$ as a function of electrolyte concentration. Ion-surface dispersion interactions are included.

charged oil-like surface due to positive adsorption of anions (and repulsion of cations), driven by their dispersion interactions. Likewise a positive potential is found at the negatively charged mica surface due to dispersion-enhanced adsorption of cations. Field reversal is commonly referred to as "charge reversal" in the literature. ${ }^{79-81}$ The phrase "charge inversion" is sometimes used to refer to field reversal. ${ }^{82,83}$ The difference between the two surfaces in the critical concentration for field reversal is because the attractive dispersion interactions of the counterion are stronger at the mica surface than at the oil-like surface, as seen in the dispersion $B$ coefficients in Table 1 .

As a consequence of field reversal (e.g., in $0.1 \mathrm{M} \mathrm{MgCl}_{2}$ ) the mica surface becomes effectively positively charged, and therefore strong adsorption of the co-ion $\mathrm{Cl}^{-}$occurs. Ion concentration profiles are shown in Figure $7 \mathrm{a}$ for surface separation $10 \AA$, with ionic dispersion interactions included. In principle a separation of $10 \AA$ is at the limits of the applicability of the Poisson-Boltzmann method, with the length scale being only ten or so times greater than the size of the ions themselves, Table 1. At this $1 \mathrm{~nm}$ scale ion-ion and ion-solvent correlations may be expected, leading to oscillatory concentration profiles which may be accounted for in a Poisson-Boltzmann calculation as a supplementary nonelectrostatic potential. ${ }^{40-42}$ In real systems these oscillations tend to be smoothed out by surface roughness at the molecular level. In any case we have omitted these higher-order effects for simplicity in order to illustrate the general impact of ion-surface dispersion interactions, which are missing in classical theory.

The effect of nonelectrostatic ionic interactions is seen most clearly in the profile of the co-ions. At the negatively charged mica surface to the right, the co-ion $\mathrm{Cl}^{-}$is positively adsorbed, driven by both attractive electrostatic forces (field reversal) and attractive ionic dispersion, both operating in the same direction. At the positively charged oil-like surface to the left, the co-ion $\mathrm{Mg}^{2+}$ is depleted (this occurs even at higher concentrations when the electrostatic potential has reversed, taking on a negative sign (Figure 6). The repulsive dispersion potential of
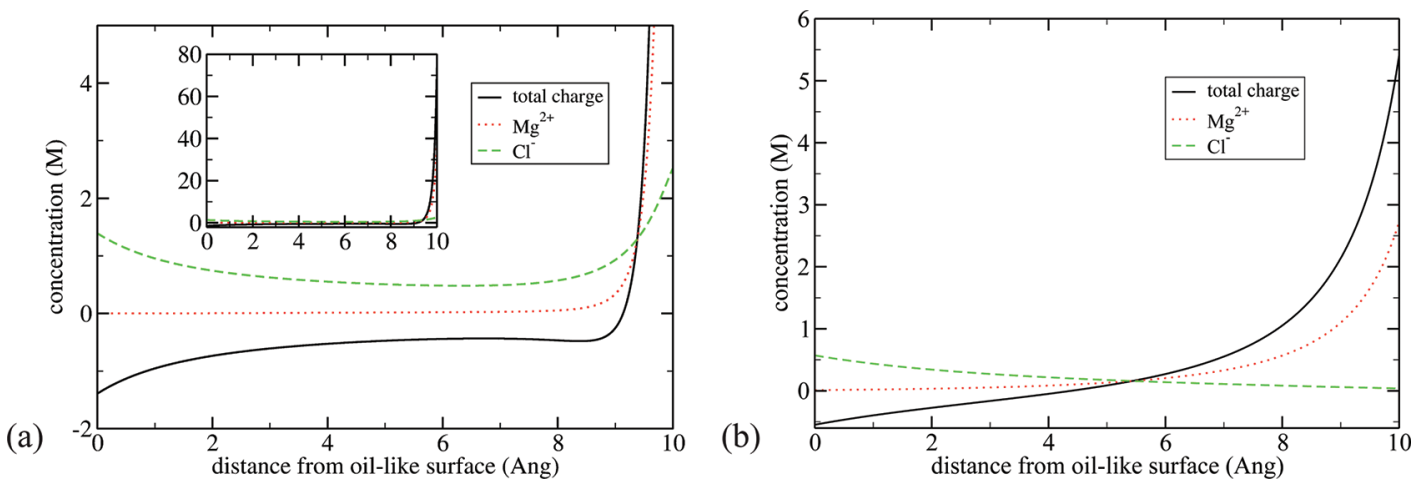

Figure 7. Charge concentration profiles for $0.1 \mathrm{M} \mathrm{MgCl}_{2}$ between a model oil-like surface on the left (tetradecane, surface charge $+0.05 \mathrm{Cm}^{-2}$ ) and mica surface on the right (surface charge $-0.1 \mathrm{Cm}^{-2}$ ), separated by $10 \AA . \mathrm{Mg}^{2+}$ and $\mathrm{Cl}^{-}$are given by the red dotted curve and green dashed curve, respectively. The total charge profile is given by the solid black curve. The inset shows the same profiles with a full range for the $\mathrm{Mg}^{2+}$ concentration at the mica surface. Shown (a) with ionic dispersion and (b) without ionic dispersion. 
$\mathrm{Mg}^{2+}$ drives depletion (in line with the underlying positive charge of the surface) even when the ions experience an attractive electrostatic force due to field reversal. The relationship between electrostatic potential and charge concentration may be counterintuitive once nonelectrostatic interactions are included.

By contrast, in the absence of ionic dispersion interactions (Figure $7 \mathrm{~b}$ ) only a relatively moderate degree of counterion adsorption takes place and no enhancement of co-ion adsorption at the mica surface is found. Consequently enhanced entropic repulsion is not predicted when nonelectrostatic ionic interactions are not included, as seen in the attractive oil-mica interaction shown in Figure 4.

A two-pronged increase therefore takes place in the repulsive entropic pressure: enhanced positive adsorption of the $\mathrm{Mg}^{2+}$ counterion is found at the mica surface because of the ionic dispersion forces of the hydrated ion. Then, more significantly, positive adsorption of the co-ion $\mathrm{Cl}^{-}$also takes place, because of the field reversal effect. The combination of both these effects leads to the strong entropic repulsion that drives oilmica separation.

3.2. Performance of Midpoint Formula. We now turn our attention away from the physical behavior of the asymmetric system and look in more detail at the role of the midpoint $z_{m}$ in the midpoint formulation. In the classical analysis of electrostatic double-layer interaction (DLVO) theory, ${ }^{53}$ the pressure has typically been taken to be the entropic term alone in the midpoint formulation, $P_{\text {en }}$ from eq 8 (together with a surfacesurface Hamaker pressure). The nonelectrostatic contribution $P_{\mathrm{NES}}$ was neglected altogether and the position of $z_{m}$ was taken at the position where $d \psi / d z=0$ (at $z_{m}=L / 2$ in the case of two identical surfaces), making $P_{\text {el-mid }}$ zero in eq $9 . P_{\text {el-surf }}$ in eq 10 was 0 under conditions of constant surface charge and canceled by the chemical contribution $P_{\text {chem }}$ in constant potential systems. It has apparently become forgotten under chargeregulated conditions. However in asymmetric systems, for instance where the two surfaces have opposite charges, there may in fact be no turning point in the electrostatic potential at all, in which case $P_{\text {el-mid }}$ in the midpoint formulation may not be dropped.

The electrostatic potential for a $3 \mathrm{M} \mathrm{MgCl}_{2}$ solution with a separation of $10 \AA ̊$ between surfaces is shown in Figure 8,

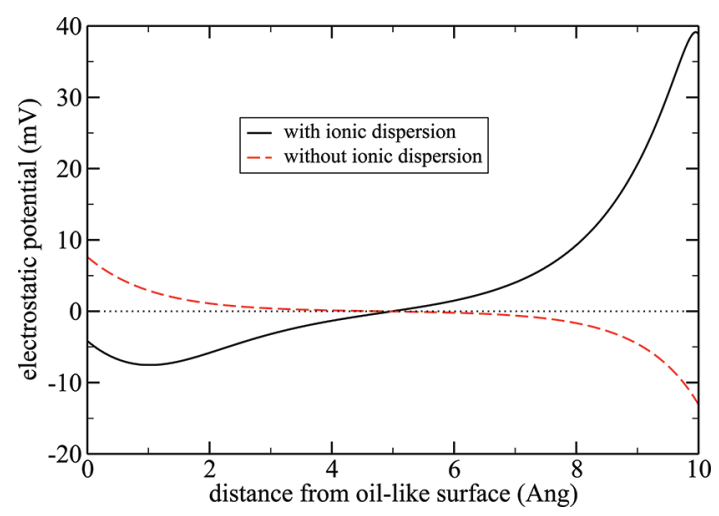

Figure 8. Electrostatic potential of $3 \mathrm{M} \mathrm{MgCl}_{2}$ between a model oillike surface on the left (tetradecane, surface charge $+0.05 \mathrm{Cm}^{-2}$ ) and mica surface on the right (surface charge $-0.1 \mathrm{Cm}^{-2}$ ), separated by $10 \AA$. The solid black curve includes ion-surface dispersion interactions, while the dashed red curve is electrostatic only (no ionic dispersion interactions). Field reversal is found at both surfaces when ionic dispersion is included. calculated with (solid black curve) and without (red dotted curve) ionic dispersion interactions. As discussed above (Figure 6) electric field reversal occurs when ionic dispersion is present. No obvious candidate for a choice of $z_{m}$ where $d \psi / d z=0$ can be determined. The slope of the potential is not 0 at the true midpoint $z_{m}=L / 2$, while two turning points related to the field reversal effect are found near near surface at $z=1 \AA$ and $z=9.9 \AA$. But the position of these turning points may vary with separation and with concentration. Rather than attempting to exclude the electrostatic term by picking $z_{m}$ where it is 0 , it is simpler in an asymmetric system to continue using the true midpoint $z_{m}=L / 2$ and explicitly include the electrostatic contribution.

But how sensitive is the pressure $P\left(L \mid z_{m}\right)$ to the choice of $z_{m}$ ? $P\left(L \mid z_{m}\right)$ (eq 7) in $3 \mathrm{M} \mathrm{MgCl}_{2}$ is shown in Figure 9 as a function

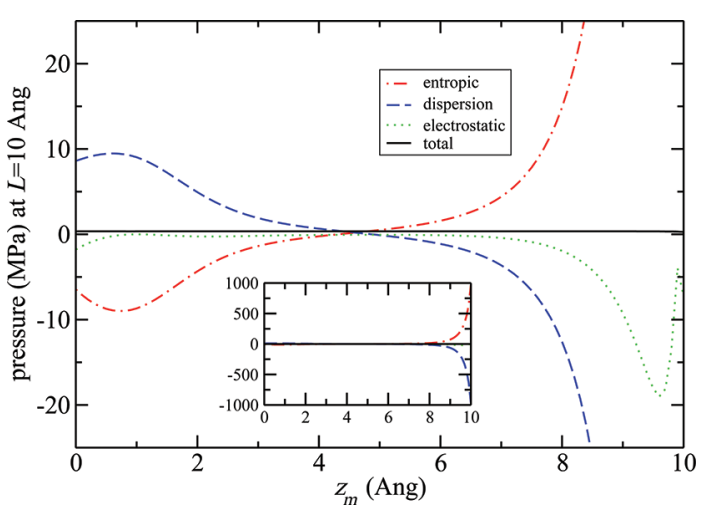

Figure 9. Pressure-distance curve $P\left(L \mid z_{m}\right)$ (excluding the Hamaker pressure) calculated using the midpoint formula, eq 7 , as a function of midpoint $z_{m}$ with fixed separation $L=10 \AA$. Shows components for $3 \mathrm{M} \mathrm{MgCl}_{2}$ electrolyte between an oil-like surface with surface charge $\sigma_{0}=+0.05$ on the left and mica $\left(\sigma_{0}=-0.1 \mathrm{Cm}^{-2}\right)$ on the right. The inset shows the full magnitude of components at high $z_{m}$. Ion-surface dispersion interactions are included. The total disjoining pressure is $0.34 \mathrm{MPa}$, independent of $z_{m}$.

of midpoint $z_{m}$ for separations $L=10$ and $L=20 \AA$. As in the example of the nonelectrolytic glucose solution in section 2.2.1, we again find that the total disjoining pressure is independent of the position of $z_{m}$. The relative magnitude of the components, however, depends strongly on $z_{m}$. The relationship between the components is therefore qualitative and can only be taken as relative, not absolute, and may only broadly distinguish attractive from repulsive components.

3.3. Charge Reversal in Monovalent Salt. We saw charge reversal (electric field reversal) in Figure 6 at $0.02 \mathrm{M} \mathrm{MgCl}_{2}$ for our mica surface and at $2 \mathrm{M} \mathrm{MgCl}_{2}$ for the oil-like surface. Charge reversal in multivalent electrolyte is not uncommon ${ }^{79-81}$ (although in some cases it may not occur where expected ${ }^{84}$ ). Charge reversal of metal oxide surfaces in monovalent electrolyte, on the other hand, is less widely reported. ${ }^{85,86}$ In Figure 10 we show the surface potentials of our model surfaces (at $10 \AA$ separation) in KI solution. Ion interaction parameters are given in Table 1. The oil-like surface has positive surface potential, matching the positive surface charge, up to a concentration of $5 \mathrm{M} \mathrm{KI}$, where it reverses to negative (the negative potential seen at very small concentrations is an unscreened negative potential originating from the opposing mica surface). The mica surface has a negative potential matching its surface charge, until field reversal takes place at $6 \mathrm{M} \mathrm{KI}$. 
It seems plausible that a colloid separation effect, similar to that described in section 3 for divalent electrolyte, may in

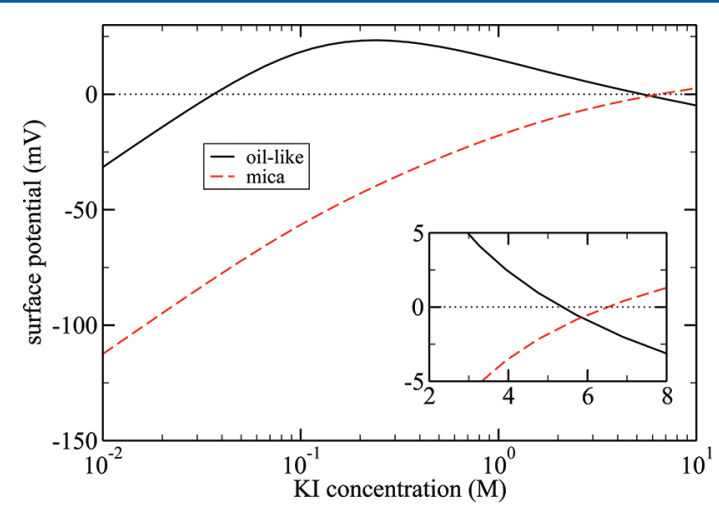

Figure 10. Electrostatic surface potentials of an oil-like surface (surface charge $\left.\sigma_{0}=+0.05\right)$ and mica $\left(\sigma_{0}=-0.1 \mathrm{Cm}^{-2}\right)$ in $\mathrm{KI}$ as a function of electrolyte concentration. Ion-surface dispersion interactions are included. The inset shows the same curves magnified at high concentration where field reversal occurs.

principle be found with monovalent electrolyte. But insofar as oil-mica repulsion depends on entropic repulsion driven by field reversal, the effect will not be found in monovalent electrolyte below $6 \mathrm{M}$ concentrations. This makes it impractical to use monovalent electrolyte to achieve separation: $6 \mathrm{M}$ concentration exceeds the solubility of many monovalent salts (KI has an uncommonly high solubility of $8 \mathrm{M}$ ).

\section{CONCLUSION}

By applying new formulas describing the ionic dispersion contribution to the disjoining pressure between dissimular surfaces, we have found a possible regime (around $0.1 \mathrm{M}$ divalent electrolyte), which may induce phase separation between oil and rock colloidal particles. It suggests applications in the stabilization or separation of heterogeneous colloidal suspensions.

We have shown that the omission of nonelectrostatic (ionic dispersion) forces from the classical theory of colloidal interactions can give seriously misleading results. For symmetric surfaces this has already been shown. Here the interpretation of direct force measurements by classical DLVO fails. The measurements and their ion specificity (Hofmeister effects) have long posed a problem That they must be included is unavoidable. It is also physically inadmissible not to do so. The present paper dealt with dissimilar surfaces, relevant to heterocoagulation an area of much practical interest. It has remained a mystery within the confines of classical electrostatic theories. We have previously evaluated ab initio dynamic polarizabilities of a whole range of ions. ${ }^{58,61,76}$ It is clear that the vast variations in ionic susceptibilities together with surface charge properties of interacting particles will cause a huge variation in forces (Hofmeister effects), inexplicable otherwise.

We have demonstrated that the traditional midpoint formulation of the disjoining pressure $P\left(L \mid z_{m}\right)$, eq 7 , is independent of the position of the midpoint $z_{m}$, even with the introduction of nonelectrostatic ionic interactions. We have also derived an alternate formulation of the disjoining pressure, eq 13, using the whole domain between the two surfaces. The two formulas for the total disjoining pressure are in agreement.

In addition to the ionic dispersion contribution to interactions between dissimilar surfaces, we have identified a term, eq 10 , belonging to the electrostatic contribution to the total disjoining pressure which arises due to a change in the surface charge as the surfaces approach each another. The term will usually provides an attractive force. This surface charge term is 0 in the case of the constant charge boundary condition and canceled by a repulsive chemical term in the case of the constant potential boundary condition. When calculating the disjoining pressure of a charge regulated system, ${ }^{38,55,87}$ however, care will need to be taken to correctly combine the surface electrostatic term and the chemical term. Additional repulsion will occur in that case from the chemical term, because of the varying surface potential.

The charge-regulated condition also needs to be re-evaluated to account for ionic dispersion interactions, in particular dispersion interactions of the hydronium ion. $\mathrm{p} K_{a}$ values used in charge regulated models have been calibrated using solely electrostatic hydronium interactions and will shift once ionic dispersion is consistently added.

The repulsive oil-rock interaction that might lead to colloid separation was found to be caused by enhanced entropic repulsion. Entropic repulsion was found to be enhanced due to positive adsorption of the co-ion at the rock surface, as a result of electric field reversal ("charge reversal"). This effect was also found in monovalent electrolye but only at impracticably high concentrations above $6 \mathrm{M}$, which exceeds the solubility of many salts.

The model used, with fixed surface charge and nonretarded dispersion interactions, was deliberately kept simple to illustrate the applicability of the new formulas. But it provides justification for further exploration with more realistic models. The effect may help explain anomalous experimental measurements observed in the interactions of crude oil with rock. ${ }^{77,78}$ Deeper subtleties to be added include charge regulation (varying surface charge and including the chemical contribution to the free energy), distances of closest approach to the surface due to finite ion sizes, image forces (including their screening), retardation effects, oscillatory ion-ion correlations ${ }^{42}$ and ion core repulsion, and, perhaps most importantly, ion cavity energies. ${ }^{27}$ Some of these may be treated using the same formalism presented in this paper, as additional nonelectrostatic ion interactions are added alongside ionic dispersion.

\section{ASSOCIATED CONTENT}

\section{S Supporting Information}

Technical details for the derivations of eqs 7 and 13 and the alternate formulas for the total disjoining pressure. This material is available free of charge via the Internet at http:// pubs.acs.org.

\section{AUTHOR INFORMATION}

\section{Corresponding Author}

*E-mail: Drew.Parsons@anu.edu.au.

\section{Notes}

The authors declare no competing financial interest.

\section{REFERENCES}

(1) Wiese, G. R.; Healy, T. W. J. Colloid Interface Sci. 1975, 52, 458467.

(2) Kihira, H.; Matijević, E. Adv. Colloid Interface Sci. 1992, 42, 1-31.

(3) Bleier, A. Colloids Surf. 1992, 66, 157-179.

(4) Islam, A. M.; Chowdhry, B. Z.; Snowden, M. J. Adv. Colloid Interface Sci. 1995, 62, 109-136.

(5) Ramakrishnan, V.; Pradip; Malghan, S. Colloids Surf., A 1998, 133, 135-142. 
(6) Garcia-Perez, P.; Pagnoux, C.; Rossignol, F.; Baumard, J.-F. Colloids Surf., A 2006, 281, 58-66.

(7) Parsons, D. F.; Boström, M.; Nostro, P. L.; Ninham, B. W. Phys. Chem. Chem. Phys. 2011, 13, 12352-12367.

(8) Ninham, B. W.; Duignan, T. T.; Parsons, D. F. Curr. Opin. Colloid Interface Sci. 2011, 16, 612-617.

(9) Borah, J. M.; Mahiuddin, S.; Sarma, N.; Parsons, D. F.; Ninham, B. W. Langmuir 2011, 27, 8710-8717.

(10) Boström, M.; Parsons, D. F.; Salis, A.; Ninham, B. W.; Monduzzi, M. Langmuir 2011, 27, 9504-9511.

(11) Salis, A.; Boström, M.; Medda, L.; Cugia, F.; Barse, B.; Parsons, D. F.; Ninham, B. W.; Monduzzi, M. Langmuir 2011, 27, 1159711604.

(12) Hogg, R.; Healy, T. W.; Fuerstenau, D. W. Trans. Faraday Soc. 1966, 62, 1638-1651.

(13) Wilson, B. A.; Crimp, M. J. Langmuir 1993, 9, 2836-2843.

(14) Truesdail, S. E.; Lukasik, J.; Farrah, S. R.; Shah, D. O.; Dickinson, R. B. J. Colloid Interface Sci. 1998, 203, 369-378.

(15) Hermansson, M. Colloids Surf., B 1999, 14, 105-119.

(16) Chang, Y.-I.; Chang, P.-K. Colloids Surf., A 2002, 211, 67-77.

(17) Biesheuvel, P. M. J. Colloid Interface Sci. 2004, 275, 514-522.

(18) Ninham, B.; Nostro, P. L. Molecular Forces and Self Assembly In Colloid, Nano Sciences and Biology; Cambridge Molecular Science; Cambridge University Press, 2010; chapter 7 coauthored with Drew Parsons.

(19) Specific Ion Effects; Kunz, W., Ed.; World Scientific: Singapore, 2010.

(20) Edwards, S. A.; Williams, D. R. M. Phys. Rev. Lett. 2004, 92, 248303.

(21) Overbeek, J. T. G. Colloids Surf. 1990, 51, 61-75.

(22) Burak, Y.; Andelman, D. J. Chem. Phys. 2001, 114, 3271-3283.

(23) Kjellander, R.; Marčelja, S. Chem. Phys. Lett. 1987, 142, 485491.

(24) Wernersson, E.; Kjellander, R. J. Chem. Phys. 2008, 129, 144701.

(25) Wernersson, E.; Kjellander, R. J. Chem. Phys. 2006, 125, 154702.

(26) Bratko, D.; Jönsson, B.; Wennerström, H. Chem. Phys. Lett.

1986, 128, 449-454.

(27) dos Santos, A. P.; Diehl, A.; Levin, Y. Langmuir 2010, 26, $10778-10783$.

(28) Ninham, B.; Yaminsky, V. Langmuir 1997, 13, 2097-2108.

(29) Koelsch, P.; Viswanath, P.; Motschmann, H.; Shapovalov, V.; Brezesinski, G.; Möhwald, H.; Horinek, D.; Netz, R. R.; Giewekemeyer, K.; Salditt, T.; Schollmeyer, H.; von Klitzing, R.; Daillant, J.; Guenoun, P. Colloids Surf., A 2007, 303, 110-136.

(30) Sloutskin, E.; Baumert, J.; Ocko, B. M.; Kuzmenko, I.; Checco, A.; Tamam, L.; Ofer, E.; Gog, T.; Gang, O.; Deutsch, M. J. Chem. Phys. 2007, 126, 054704

(31) Brown, M. A.; Winter, B.; Faubel, M.; Hemminger, J. C. J. Am. Chem. Soc. 2009, 131, 8354-8355.

(32) Otten, D. E.; Shaffer, P. R.; Geissler, P. L.; Saykally, R. J. Proc. Natl. Acad. Sci. U. S. A. 2012, 109, 701-705.

(33) Jungwirth, P.; Tobias, D. Chem. Rev. 2006, 106, 1259-1281.

(34) Vrbka, L.; Mucha, M.; Minofar, B.; Jungwirth, P.; Brown, E. C.; Tobias, D. J. Curr. Opin. Colloid Interface Sci. 2004, 9, 67-73.

(35) Warren, G. L.; Patel, S. J. Phys. Chem. C 2008, 112, 7455-7467.

(36) Ottosson, N.; Heyda, J.; Wernersson, E.; Pokapanich, W.; Svensson, S.; Winter, B.; Ohrwall, G.; Jungwirth, P.; Bjorneholm, O. Phys. Chem. Chem. Phys. 2010, 12, 10693-10700.

(37) Howard, J. J.; Perkyns, J. S.; Pettitt, B. M. J. Phys. Chem. B 2010, 114, 6074-6083, PMID: 20405885

(38) Boström, M.; Williams, D. R. M.; Ninham, B. W. Langmuir 2002, 18, 8609-8615.

(39) Ruckenstein, E.; Manciu, M. Adv. Colloid Interface Sci. 2003, 105, 177-200.

(40) Vrbka, L.; Lund, M.; Kalcher, I.; Dzubiella, J.; Netz, R. R.; Kunz, W. J. Chem. Phys. 2009, 131, 154109.

(41) Schwierz, N.; Horinek, D.; Netz, R. R. Langmuir 2010, 26, $7370-7379$.

(42) Marčelja, S. Curr. Opin. Colloid Interface Sci. 2011, 16, 579-583.
(43) Payens, T. A. J. Philips Res. Rep. 1955, 10, 425-481.

(44) Bell, G.; Levine, S.; Stephens, D. J. Colloid Interface Sci. 1972, 38, 609-615.

(45) Chan, D. Y. C.; Mitchell, D. J. J. Colloid Interface Sci. 1983, 95, 193-197.

(46) Borkovec, M.; Behrens, S. H. J. Phys. Chem. B 2008, 112, 10795-10799.

(47) Derjaguin, B. V. Adv. Colloid Interface Sci. 1985, 22, 299-303.

(48) Chan, D. Y. C.; Pashley, R. M.; White, L. R. J. Colloid Interface Sci. 1980, 77, 283-285.

(49) Glendinning, A. B.; Russel, W. B. J. Colloid Interface Sci. 1983, 93, 95-104.

(50) Sciortino, F.; Tartaglia, P.; Zaccarelli, E. J. Phys. Chem. B 2005, 109, 21942-21953.

(51) Zhang, H.; Liu, Y.; Zhang, J.; Wang, C.; Li, M.; Yang, B. J. Phys. Chem. C 2008, 112, 1885-1889.

(52) Olsen, S. N.; Andersen, K. B.; Randolph, T. W.; Carpenter, J. F.; Westh, P. Biochim. Biophys. Acta 2009, 1794, 1058-1065.

(53) Israelachvili, J. N. Intermolecular and Surface Forces, 2nd ed.; Academic Press: London, 1991.

(54) Ninham, B. W.; Parsegian, V. A. J. Theor. Biol. 1971, 31, 405428 .

(55) Chan, D.; Perram, J.; White, L.; Healy, T. J. Chem. Soc., Faraday Trans. 1 1975, 71, 1046-1057.

(56) Hall, D. G. J. Colloid Interface Sci. 1985, 108, 411-413.

(57) Overbeek, J. T. G. In Colloid Science; Elsevier: Amsterdam, 1952; Vol. I, Chapter VI. The Interaction Between Colloidal Particles, pp 245-277.

(58) Parsons, D. F.; Ninham, B. W. Langmuir 2010, 26, 1816-1823.

(59) Adamo, C.; Cossi, M.; Scalmani, G.; Barone, V. Chem. Phys. Lett. 1999, 307, 265-271.

(60) Thom H. Dunning, J. J. Chem. Phys. 1989, 90, 1007-1023.

(61) Parsons, D. F.; Ninham, B. W. J. Phys. Chem. A 2009, 113, $1141-1150$.

(62) Parsegian, V. A.; Weiss, G. H. J. Colloid Interface Sci. 1981, 81, 285-289.

(63) Mahanty, J.; Ninham, B. W. J. Chem. Soc., Faraday Trans. 2 1975, 71, 119-137.

(64) Mahanty, J.; Ninham, B. W. Dispersion Forces; Academic Press: London, 1976.

(65) Buckley, J. S.; Takamura, K.; Morrow, N. R. SPE Reservoir Eng. $1989,4,332-340$.

(66) Boström, M.; Lima, E. R. A.; Biscaia, E. C.; Tavares, F. W.; Nostro, P. L.; Parsons, D. F.; Deniz, V.; Ninham, B. W. J. Phys. Chem. B 2009, 113, 8124-8127.

(67) Parsons, D. F.; Boström, M.; Maceina, T. J.; Salis, A.; Ninham, B. W. Langmuir 2010, 26, 3323-3328.

(68) Boström, M.; Williams, D. R. M.; Ninham, B. W. Langmuir 2001, 17, 4475-4478.

(69) Bostrom, M.; Williams, D.; Ninham, B. Langmuir 2002, 18, 6010-6014.

(70) Boström, M.; Ninham, B. W. Langmuir 2004, 20, 7569-7574.

(71) Kunz, W.; Belloni, L.; Bernard, O.; Ninham, B. J. Phys. Chem. B 2004, 108, 2398-2404.

(72) Martín-Molina, A.; Ibarra-Armenta, J. G.; Quesada-Pérez, M. J. Phys. Chem. B 2009, 113, 2414-2421.

(73) Chan, D.; Richmond, P. Proc. R. Soc. London, Ser. A 1977, 353, $163-176$

(74) Dagastine, R. R.; Prieve, D. C.; White, L. R. J. Colloid Interface Sci. 2000, 231, 351-358.

(75) Marcus, Y. Pure Appl. Chem. 1987, 59, 1093-1101.

(76) Parsons, D. F.; Ninham, B. W. Langmuir 2010, 26, 6430-6436.

(77) Liu, L.; Buckley, J. S. J. Pet. Sci. Eng. 1999, 24, 75-83.

(78) Shedid, S. A.; Ghannam, M. T. J. Pet. Sci. Eng. 2004, 44, 193203.

(79) Kékicheff, P.; Marčelja, S.; Senden, T. J.; Shubin, V. E. J. Chem. Phys. 1993, 99, 6098-6113.

(80) Chakir, A.; Bessiere, J.; Kacemi, K. E.; Marouf, B. J. Hazard. Mater. 2002, 95, 29-46. 
(81) Alkan, M.; Demirbas, Ö.; Doğan, M. Microporous Mesoporous Mater. 2005, 84, 192-200.

(82) Grosberg, A. Y.; Nguyen, T. T.; Shklovskii, B. I. Rev. Mod. Phys. 2002, 74, 329-345.

(83) Jiménez-Ángeles, F.; Lozada-Cassou, M. J. Chem. Phys. 2008, 128, 174701.

(84) Pochard, I.; Denoyel, R.; Couchot, P.; Foissy, A. J. Colloid Interface Sci. 2002, 255, 27-35.

(85) Kosmulski, M.; Rosenholm, J. B. Adv. Colloid Interface Sci. 2004, 112, 93-107.

(86) Chassagne, C.; Mietta, F.; Winterwerp, J. J. Colloid Interface Sci. 2009, 336, 352-359.

(87) Miklavic, S. J.; Ninham, B. W. J. Colloid Interface Sci. 1990, 134, 305-311. 